\title{
Generating free redundancy XML documents from non normalized relational views using a statistically approach
}

\begin{abstract}
Converting relational database into XML is increasing daily for publishing and exchanging the business applications data on the Web. Most of the current approaches and products convert the non normalized relational views to XML documents using flat-based that causes data redundancy which leads to generate a massive data. This paper proposes an approach to reduce the redundancy for the XML documents that are generated from non normalized relational views. The proposed approach includes two steps: the first is extracting the nested view from the flat relational view using a statistically approach for counting and removing the data redundancy. The statistically approach is based on the analysis of functional dependency taking into account the frequency of the data values for the columns of the relational views. The document based on the blocks that are created according to frequency of the data values for the first column which has the most frequent of the data values.
\end{abstract}

Keyword: Blocks; Frequency; Groups; Nested view; Nested XML documents; Non normalized relational view; Redundancy; XML 